\title{
Oxidation of acyclic alkenes and allyl and benzyl ethers with $\mathrm{DIB} / \mathrm{t} \mathrm{BuOOH} / \mathrm{Mg}(\mathrm{OAc})_{2}$
}

\author{
Thanapat Sastraruji ${ }^{\mathrm{a}}$, Stephen G. Pyne ${ }^{\mathrm{a}}$ * and Alison T. Ung ${ }^{\mathrm{b}}$ \\ ${ }^{a}$ School of Chemistry, University of Wollongong, Wollongong, New South Wales, 2522, Australia \\ ${ }^{b}$ Department of Chemistry and Forensic Science, University of Technology Sydney, Sydney, 2007, Australia
}

\section{ARTICLE INFO}

\section{ABSTRACT}

\section{Article history:}

Received

Received in revised form

Accepted

Available online

Keywords:

Allylic oxidation

Oxidative clevage

acyclic alkene

benzyl ether

allyl ether
Oxidation of (11Z)-1',2'-didehydrostemofoline with DIB/TBHP/ $\mathrm{Mg}(\mathrm{OAc})_{2} \bullet 4 \mathrm{H}_{2} \mathrm{O}$ resulted in oxidative cleavage of the $\mathrm{C}-11-\mathrm{C}-12$ double bond instead of the desired allylic oxidation of the 1-butenyl side chain. Stemofoline gave a similar result. The oxidation of more simple terminal alkenes was regioselective and gave vinyl ketones while allyl and benzyl ethers gave acrylate and benzoate esters, respectively. Allyl and benzyl ethers could be chemoselectively oxidized in the presence of a terminal alkene or benzyl group. Oxidation of an internal alkene was poorly regioselective, in contrast to the oxidation of 1-substituted cyclohexenes.

\section{Introduction}

In 2010, Zhao and Yeung reported the regioselective allylic oxidation of 1-substituted cyclohexenes $\mathbf{1}$ to 3 -substituted cyclohexanones 2 using diacetoxyiodobenzene (DIB), tert-butyl hydroperoxide (TBHP) and $\mathrm{Mg}(\mathrm{OAc})_{2} \bullet 4 \mathrm{H}_{2} \mathrm{O}$ in an ester solvent (Scheme 1). ${ }^{1} n$-Butyl butanoate was found to be the solvent of choice.

In connection with our studies to prepare rare Stemona alkaloids and analogues for biological studies, ${ }^{2}$ starting from (11Z)-1', $2^{\prime}$-didehydrostemofoline $\mathbf{3}$, we examined the reaction of 3 with $\mathrm{DIB} / \mathrm{TBHP} / \mathrm{Mg}(\mathrm{OAc})_{2} \bullet 4 \mathrm{H}_{2} \mathrm{O}$ with the desire to directly prepare the known enone 4 (Scheme 2). This paper reports the results of this study and related oxidation reactions on stemofoline, more simple acyclic alkenes and allyl and benzyl ethers.
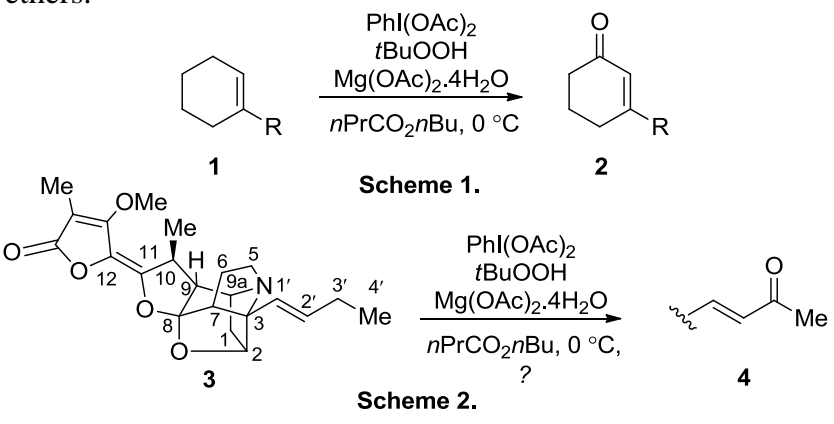

\section{Results and discussion}

\subsection{Oxidation of (11Z)-1',2'-didehydrostemofoline 3}

With the aim of preparing enone 4 (Scheme 2), (11Z)-1', $2^{\prime}$ didehydrostemofoline $\mathbf{3}$ was treated under the oxidation

* Corresponding author. Tel.: +61 24221 3511; fax: +61 24221 4287; e-mail: spyne@uow.edu.au conditions reported by Zhao and Yeung. ${ }^{1}$ Treatment of $\mathbf{3}$ in $n$ butyl butanoate with DIB (1 equiv.)/TBHP (3 equiv.)/ $\mathrm{Mg}(\mathrm{OAc})_{2} \bullet 4 \mathrm{H}_{2} \mathrm{O}$ (1 equiv.) at $0{ }^{\circ} \mathrm{C}$ for $15 \mathrm{~h}$ gave not the enone 4 but a mixture of the lactone 5 and enone $\mathbf{6}$, a result of overall oxidative cleavage of the C-11-C-12 double bond of $\mathbf{3}$ and further side chain allylic oxidation of 5 to give $\mathbf{6}$ (Scheme 3 ). Purification of this mixture by column chromatography gave $\mathbf{5}$ and $\mathbf{6}$ in yields of 51 and 13\%, respectively. Treatment of $\mathbf{5}$ under the same oxidative conditions gave only $20 \%$ yield of enone $\mathbf{6}$. To improve the yield of enone $\mathbf{6}$ from $\mathbf{5}$, the stoichiometric ratios of the reactants were systematically varied. The optimum conditions found were DIB (8 equiv.)/TBHP (24 equiv.)/ $\mathrm{Mg}(\mathrm{OAc})_{2} \bullet 4 \mathrm{H}_{2} \mathrm{O}$ (1 equiv.) at $0{ }^{\circ} \mathrm{C}$ for $15 \mathrm{~h}$. Under these conditions the yield of enone 6 was improved to $42 \%$. The corresponding $\mathrm{N}$-oxides of $\mathbf{5}$ or $\mathbf{6}$ were not detected.

\subsection{Oxidation of stemofoline 7}

Under similar conditions to those described above for the oxidation of $\mathbf{3}$, stemofoline $\mathbf{7}$ was converted to the known lactone $\mathbf{8}$ in 58\% yield (Scheme 4). This compound was prepared previously by us by first osmium-catalyzed dihydroxylation of the C-11-C-12 double bond of 7 followed by oxidative cleavage of the result diol with $\mathrm{NaIO}_{4}$ in $34 \%$ overall yield. ${ }^{2}$
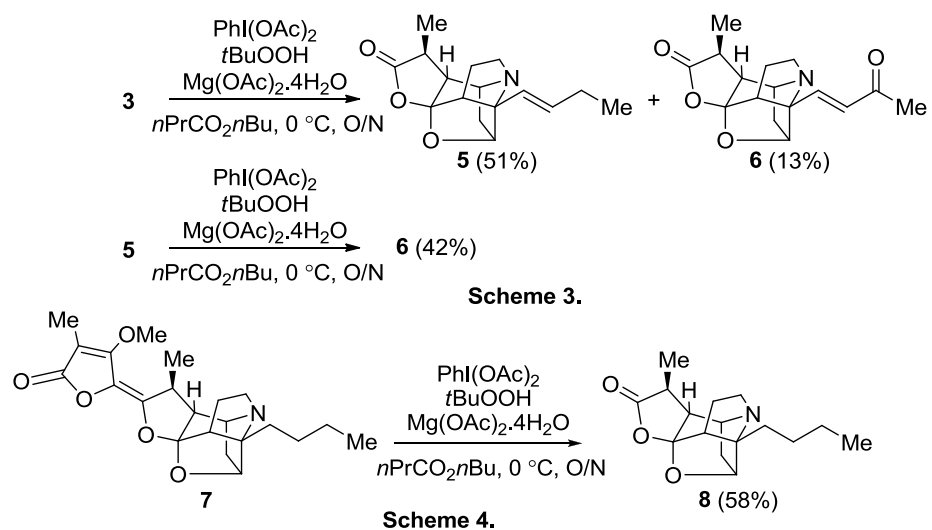


\subsection{Oxidation of simple acyclic alkenes}

To further examine the potential of these oxidation conditions the allylic oxidations of simpler acyclic alkenes were studied. The results of these studies are shown in Table 1. Hex-5-enyl benzoate 9 was used as model compound to optimize the reaction conditions, which were then applied to the allylic oxidation of the other alkenes 10-12 shown in Table 1. The optimum reaction conditions were found to be identical to those reported by Zhao and Yeung. ${ }^{1}$ (DIB (1 equiv.)/TBHP (3 equiv.) $/ \mathrm{Mg}(\mathrm{OAc})_{2} \bullet 4 \mathrm{H}_{2} \mathrm{O}$ ( 1 equiv.) at $0{ }^{\circ} \mathrm{C}$ in $n$-butyl butanoate. The allylic oxidation reactions of the terminal alkenes $\mathbf{9}$ and $\mathbf{1 0}$ were regioselective and proceeded to provide the vinyl ketones 9a and 10a, respectively. The higher yield of enone 9a over 10a may be a result of formation of an intermediate involving coordination of the benzoyl ester carbonyl and the hypervalent iodine intermediate $\left(\mathrm{PhI}(\mathrm{OOtBu})_{2}\right)$. This intermediate may be able to more readily abstract, intramolecularly, the allylic $\mathrm{H}$-atom in the shorter chain substrate 9. ${ }^{3}$ The oxidation reaction of the internal alkene $\mathbf{1 1}$ was not regioselective and gave a mixture of three enones (9a, 11a and 11b). Based on their isolated yields, about one third of the product (9a) resulted from initial oxidation at the terminal methyl group and two thirds of the products $(\mathbf{1 1 a}, \mathbf{b})$ from initial oxidation at the internal allylic methylene group.

Table 1. Allylic oxidation of Substrates
Oxidation of the alkene 12, gave the alkene $\mathbf{9}$ as the major product resulting from chemoselective oxidation of the benzyl ether group of $\mathbf{1 2}$ to a benzoate. Small amounts of doubly oxidized product $(\mathbf{9 a})$ and the enone $12 \mathbf{a}$ were also isolated.

For the oxidations of substrates 13-18 the products were difficult to separate from the solvent $n$-butyl butanoate. Thus, ethyl acetate was used as alternative ester solvent. When allyl ether 13 was treated with DIB (1 equiv.)/TBHP (3 equiv.)/ $\mathrm{Mg}(\mathrm{OAc})_{2} \bullet 4 \mathrm{H}_{2} \mathrm{O}$ (1 equiv.) at $0{ }^{\circ} \mathrm{C}$ in ethyl acetate the yield of the acrylate ester 13a was only $67 \%$. Therefore, further optimization of the reaction conditions were carried out. It was found that by using DIB (1.5 equiv.)/TBHP (4.5 equiv.)/ $\mathrm{Mg}(\mathrm{OAc})_{2} \cdot 4 \mathrm{H}_{2} \mathrm{O}$ (1 equiv.) at $0{ }^{\circ} \mathrm{C}$ in ethyl acetate, the yield of 13a was increased to $90 \%$. This reaction was highly chemoselective and no products arising from benzylic oxidation of 13 or 13a could be isolated. The same oxidation conditions when applied to the benzyl ether $\mathbf{1 4}$ gave, chemoselectively, the benzoate $\mathbf{1 4 a}$ in $95 \%$ yield. For the substrates $\mathbf{1 5}-\mathbf{1 8}$ the optimum conditions were found to be DIB (1.5 equiv.)/TBHP (6 equiv.) $/ \mathrm{Mg}(\mathrm{OAc})_{2} \bullet 4 \mathrm{H}_{2} \mathrm{O}$ ( 1 equiv.). These conditions gave the benzoate ester 15a and the acrylate esters 16a and 17a in improved yields of 93, 91 and $88 \%$, respectively over those using these reagents in a 1:3:1: molar equivalent ratio. The oxidation reaction of $\mathbf{1 8}$ was not chemoselective and gave a mixture of the esters $18 \mathbf{a}$ and $\mathbf{1 8 b}$ and the diester18c.

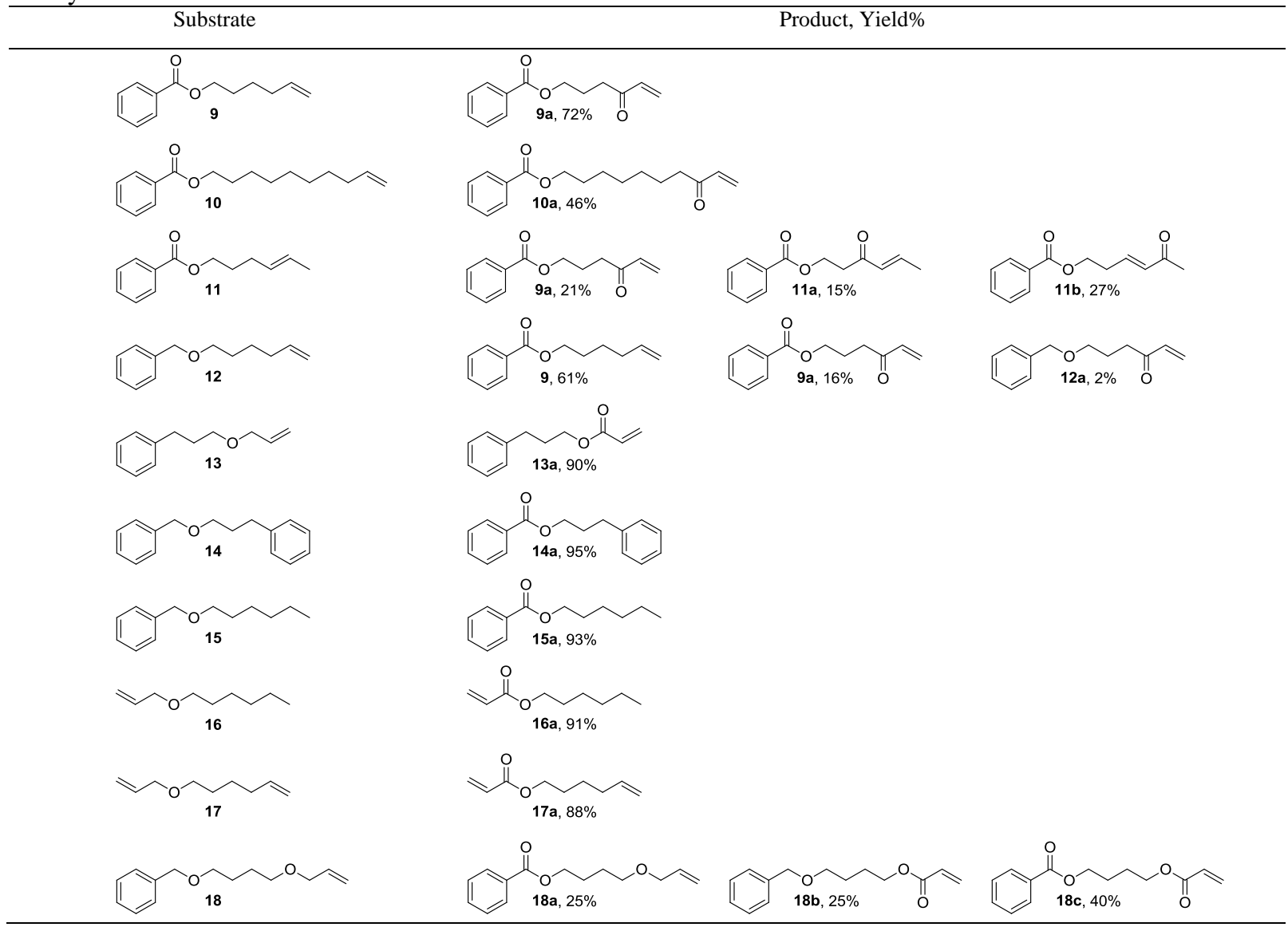

\section{Conclusions}

In conclusion, oxidation of $(11 Z)-1^{\prime}, 2^{\prime}$-didehydrostemofoline 3 with DIB/TBHP/Mg(OAc $)_{2} \cdot 4 \mathrm{H}_{2} \mathrm{O}$ resulted in oxidative cleavage of the $\mathrm{C}-11-\mathrm{C}-12$ double bond instead of the desired allylic oxidation at the 1-butenyl side chain. Stemofoline 7 gave a similar result under identical oxidation conditions. The oxidation of more simple terminal alkenes was regioselective and gave vinyl ketones while allyl and benzyl ethers gave acrylate and benzoate esters, respectively. Allyl and benzyl ethers could be chemoselectively oxidized in the presence of a terminal alkene or benzyl group. Oxidation of an internal alkene was poorly regioselective, in contrast to the oxidation of 1-substituted cyclohexenes. ${ }^{1}$ These studies further demonstrate the usefulness 
and limitations of this relatively straight forward oxidation system to acyclic alkenes and benzyl and allyl ethers.

\section{Experimental section}

\subsection{General}

All ${ }^{1} \mathrm{H}$ NMR (500 MHz) and ${ }^{13} \mathrm{C}$ NMR (125 MHz) spectra were determined in $\mathrm{CDCl}_{3}$ solution. ${ }^{1} \mathrm{H}$ NMR assignments were achieved with the aid of gCOSY and, in some cases, NOESY experiments. ${ }^{13} \mathrm{C}$ NMR assignments were based upon DEPT, gHSQC, and gHMBC experiments. Electrospray (ESI) mass spectra were obtained on a VG Autospec spectrometer. Highresolution mass spectra (HRMS) were determined on a micromass QTof2 spectrometer using polyethylene glycol or polypropylene glycol as the internal standard. Optical rotations were measured using a $1 \mathrm{~cm}$ cell, in a Jasco DIP-370 digital polarimeter or a $10 \mathrm{~cm}$ cell, in a Jasco P-2000 polarimeter. Infrared spectra were obtained as neat samples on a Shimadzu MIRacle 10 FTIR by the single reflection ATR method.

\subsubsection{Starting materials}

The known starting material $\mathbf{3}$ and $\mathbf{7}$ was isolated from an unidentified Stemona species that we reported earlier. ${ }^{4}$ All other chemicals were purchased from commercial suppliers and were used without further purification. Dec-9-enyl benzoate $\mathbf{1 0}$ and (E)-hex-4-enyl benzoate $\mathbf{1 1}$ were prepared according to a literature procedures from benzoylation reactions of the commercially available alcohols. ${ }^{5}$ Benzyl-5-hexenyl ether 12, allyl-5-hexenyl ether 13, benzyl-3-phenylpropyl ether 14, benzyl hexyl ether 15, allyoxy-3-propyl benzene 16, allyl hexyl ether 17 and ((4-(allyloxy) butoxy) methyl)benzene 18 were prepared according to literature procedures from benzylation or allylation reactions of the commercially available alcohols. ${ }^{6}$ Spectroscopic data of $12^{7}, \mathbf{1 3}^{8}, \mathbf{1 4}^{9}, \mathbf{1 5}^{10}, \mathbf{1 6}^{11}$ and $\mathbf{1 8}^{8}$ was identical to those reported.

Dec-9-enyl benzoate 10: colourless oil; IR $v_{\max } 2927,2855$, $1719,1270,1111 \mathrm{~cm}^{-1} ;{ }^{1} \mathrm{H}$ NMR $\delta 8.04(\mathrm{~d}, J=7.0 \mathrm{~Hz}, 2 \mathrm{H}$, ArH2 and 6), $7.54(\mathrm{t}, J=7.0 \mathrm{~Hz}, 1 \mathrm{H}, \mathrm{ArH} 4), 7.43(\mathrm{t}, J=7.0 \mathrm{~Hz}, 2 \mathrm{H}$, ArH3 and 5), 5.82-5.77 (m, 1H, H9'), 4.99 (d, $J=17.0 \mathrm{~Hz}, 1 \mathrm{H}$, H10'a), 4.93 (d, $\left.J=10.0 \mathrm{~Hz}, 1 \mathrm{H}, \mathrm{H} 10^{\prime} \mathrm{b}\right), 4.31$ (t, $J=7.0 \mathrm{~Hz}, 2 \mathrm{H}$, $\mathrm{H} 1^{\prime}$ ), 2.04 (dt, $\left.J=7.0,7.0 \mathrm{~Hz}, 2 \mathrm{H}, \mathrm{H} 8^{\prime}\right), 1.77$ (tt, $J=7.0,7.0 \mathrm{~Hz}$, $\left.2 \mathrm{H}, \mathrm{H} 2^{\prime}\right), 1.44$ (dt, $\left.J=7.0,7.0 \mathrm{~Hz}, 2 \mathrm{H}, \mathrm{H} 3^{\prime}\right), 1.41-1.32(\mathrm{~m}, 8 \mathrm{H}$, $\mathrm{H}^{\prime}, 5^{\prime}, 6^{\prime}$ and $\left.7^{\prime}\right) ;{ }^{13} \mathrm{C}$ NMR $\delta 166.7$ (CO), $139.2\left(\mathrm{C}^{\prime}\right), 132.9$ (ArC4), 130.7 (ArC1), 129.6 ( $\mathrm{ArC} 2$ and 6), 128.4 (ArC3 and 5), $114.2\left(\mathrm{C} 10^{\prime}\right), 65.2\left(\mathrm{C} 1^{\prime}\right), 33.9\left(\mathrm{C}^{\prime}\right), 29.5\left(\mathrm{C}^{\prime}\right), 29.3\left(\mathrm{C}^{\prime}\right), 29.1$ (C6'), 29.0 (C5'), $28.8\left(\mathrm{C} 4^{\prime}\right), 26.1$ (C3'); HRESIMS m/z 261.1846 $[\mathrm{MH}]^{+}$, calcd for $\mathrm{C}_{17} \mathrm{H}_{25} \mathrm{O}_{2} 261.1855$.

(E)-Hex-4-enyl benzoate 11: colourless oil; IR $v_{\max } 2936$, 2855, 1717, 1269, $1110 \mathrm{~cm}^{-1} ;{ }^{1} \mathrm{H}$ NMR $\delta 8.04(\mathrm{~d}, J=7.0 \mathrm{~Hz}, 2 \mathrm{H}$, ArH2 and 6), 7.55 (t, $J=7.0 \mathrm{~Hz}, 1 \mathrm{H}, \operatorname{ArH} 4), 7.44(\mathrm{t}, J=7.0 \mathrm{~Hz}$, $2 \mathrm{H}, \mathrm{ArH} 3$ and 5), 5.50-5.40 (m, 2H, $\mathrm{H} 4^{\prime}$ and $\left.5^{\prime}\right), 4.32(\mathrm{t}, J=7.0$ $\mathrm{Hz}, 2 \mathrm{H}, \mathrm{H} 1^{\prime}$ ), 2.14 (dt, $J=7.0,7.0 \mathrm{~Hz}, 2 \mathrm{H}, \mathrm{H} 3^{\prime}$ ), 1.83 (tt, $J=7.0$, $\left.7.0 \mathrm{~Hz}, 2 \mathrm{H}, \mathrm{H} 2^{\prime}\right), 1.65\left(\mathrm{~d}, J=7.0 \mathrm{~Hz}, 3 \mathrm{H}, \mathrm{H6}{ }^{\prime}\right) ;{ }^{13} \mathrm{C}$ NMR $\delta 166.8$ (COO), 132.9 (ArC4), 130.6 (ArC1), 130.1 (C4'), 129.7 (ArC2 and 6), 128.5 (ArC3 and 5), $126.0\left(\mathrm{C}^{\prime}\right), 64.6\left(\mathrm{C1}^{\prime}\right), 29.1\left(\mathrm{C} 3^{\prime}\right)$, $28.7\left(\mathrm{C}^{\prime}\right), 18.0\left(\mathrm{C6}^{\prime}\right)$; HRESIMS $\mathrm{m} / \mathrm{z}$ 205.1237 [MH] ${ }^{+}$, calcd for $\mathrm{C}_{13} \mathrm{H}_{17} \mathrm{O}_{2} 205.1229$.

Allyl-5-hexenyl ether 17: colourless oil; IR $v_{\max } 2925,2855$, 1104, 993, $909 \mathrm{~cm}^{-1}$; ${ }^{1} \mathrm{H}$ NMR $\delta{ }^{1} \mathrm{H}$ NMR $\delta$ 5.95-5.87 $(\mathrm{m}, 1 \mathrm{H}$, H2), 5.85-5.76 (m, 1H, H5'), 5.26 (d, $J=17.5,1 \mathrm{H}, \mathrm{H} 1 \mathrm{a}), 5.16$ (d, $J=10.0,1 \mathrm{H}, \mathrm{H} 1 \mathrm{~b}), 5.00$ (d, $\left.J=17.5,1 \mathrm{H}, \mathrm{H} 6^{\prime} \mathrm{a}\right), 4.94$ (d, $J=$ $10.5 \mathrm{~Hz}, 1 \mathrm{H}, \mathrm{H6}^{\prime} \mathrm{b}$ ), 3.96 (d, $\left.J=5.5 \mathrm{~Hz}, 1 \mathrm{H}, \mathrm{H} 3\right), 3.43$ (t, $J=7.5$ $\left.\mathrm{Hz}, 1 \mathrm{H}, \mathrm{H} 1^{\prime}\right), 2.07$ (dt, $\left.J=7.5,7.5 \mathrm{~Hz}, 1 \mathrm{H}, \mathrm{H} 4^{\prime}\right), 1.61$ (tt, $J=7.5$, $\left.7.5 \mathrm{~Hz}, 1 \mathrm{H}, \mathrm{H} 2^{\prime}\right), 1.46\left(\mathrm{tt}, J=7.5,7.5 \mathrm{~Hz}, 1 \mathrm{H}, \mathrm{H} 3{ }^{\prime}\right) ;{ }^{13} \mathrm{C} \mathrm{NMR} \delta$ 138.8 (C5), 135.2 (C2), 116.7 (C1), 114.6 (C6'), 71.9 (C3), 70.3 (C1'), $33.7\left(\mathrm{C}^{\prime}\right), 29.3$ (C2'), 25.6 (C3'); HRESIMS was non detectable.

\subsection{General procedure for the DIB/TBHP protocol}

To a solution of the substrate in n-butyl butanoate was added DIB ( $1-1.5$ equiv.) and $\mathrm{Mg}(\mathrm{OAc})_{2} \bullet 4 \mathrm{H}_{2} \mathrm{O}$ (1 equiv.) at $0{ }^{\circ} \mathrm{C}$. The resulting suspension was vigorously stirred and a solution of TBHP $(6.0 \mathrm{M}$ in decane, $3-6$ equiv.) was added. The solution was further stirred overnight at $0{ }^{\circ} \mathrm{C}$ (total consumption of starting material as indicated by TLC analysis) and then filtered. Diethyl ether was added to the filtrate $(5 \mathrm{~mL})$ and the solutiuon was washed with water $(5 \mathrm{~mL})$. The solution was dried $\left(\mathrm{MgSO}_{4}\right)$, evaporated and the residue was purified by flash column chromatography on silica gel using gradient or isocratic elution.

4.2.1. $\quad(2 S, 2 a R, 6 S, 7 a S, 7 b S, 8 R, 9 S)-7 b-(1-$ butenyl)hexahydro-9methyl-4H-2,2,6-(epoxy[1]propanyl[3]ylidene)furo[2,3,4-gh] pyrrolizin-10-one (5) and (2S,2aR,6S,7aS,7bS,8R,9S)-7b-[(1E)buten-3-onyl]hexahydro-9-methyl-4H-2,2,6-(epoxy[1]propanyl [3]ylidene)furo[2,3,4-gh]pyrrolizin-10-one (6)

The title compounds were obtained using the general procedure described above from $3(80.4 \mathrm{mg}, 0.21 \mathrm{mmol}), n$-butyl butanoate $(1 \mathrm{~mL}), \mathrm{DIB}(67.3 \mathrm{mg}, 0.21 \mathrm{mmol}), \mathrm{Mg}(\mathrm{OAc})_{2} \bullet 4 \mathrm{H}_{2} \mathrm{O}$ (44.8 mg, $0.21 \mathrm{mmol}$ ) and TBHP (125.4 $\mu \mathrm{L}, 0.63 \mathrm{mmol})$. Separation of the crude reaction products by column chromatography using gradient elution from $100 \%$ EtOAc to $\mathrm{MeOH} / \mathrm{EtOAc}(1: 4)$ gave $29.0 \mathrm{mg}(51 \%)$ of $\mathbf{5}$ and $8.0 \mathrm{mg}(13 \%)$ of 6 .

5: Pale yellow gum; $[\alpha]_{D}^{25}+23\left(c 2.3, \mathrm{CHCl}_{3}\right)$; IR $v_{\max } 2966$, 2885, 1786, 1664, 1021, $968 \mathrm{~cm}^{-1} ;{ }^{1} \mathrm{H}$ NMR $\delta 5.79(\mathrm{dt}, J=15.5$, $6.3 \mathrm{~Hz}, 1 \mathrm{H}, \mathrm{H}-2^{\prime}$ ), 5.49 (d, J=15.5 Hz, 1H, H-1'), 4.29 (br s, $1 \mathrm{H}$, H-2), 3.47 (br s, 1H, H-9a), 3.13 (ddd, $J=15.5,10.5,5.5 \mathrm{~Hz}, 1 \mathrm{H}$, H-5a), 3.02 (ddd, $J=13.0,8.5,5.0 \mathrm{~Hz}, 1 \mathrm{H}, \mathrm{H}-5 \mathrm{~b}$ ), 2.84 (d, $J=$ $6.0 \mathrm{~Hz}, 1 \mathrm{H}, \mathrm{H}-7), 2.79$ (dq, 1H, $J=11.5,7.0, \mathrm{H}-10), 2.11-2.06$ (m, 1H, H-3') 1.94-1.89 (m, 1H, H-1a), 1.93-1.88 (m, 1H, H9), 1.86-1.79 (m, 1H, H-6a), 1.80-1.74 (m, 1H, H-1b), 1.77-1.70 (m, 1H, H-6b), 1.27 (d, $J=7.5 \mathrm{~Hz}, 3 \mathrm{H}, \mathrm{H}-12), 1.00$ (t, $\left.J=7.5 \mathrm{~Hz}, 3 \mathrm{H}, \mathrm{H}-4^{\prime}\right) ;{ }^{13} \mathrm{C}$ NMR $\delta 178.4(\mathrm{C}-11), 133.6\left(\mathrm{C}-2^{\prime}\right)$, 126.6 (C-1'), 109.3 (C-8), 83.4 (C-3), 81.0 (C-2), 61.1 (C-9a), 51.4 (C-7), 45.8 (C-9), 48.2 (C-5), 35.9 (C-10), 32.5 (C-1), 26.9 (C-6), 25.5 (C-3'), 13.6 (C-4'), 13.4 (C-12); HRESIMS $\mathrm{m} / \mathrm{z}$ $276.1604[\mathrm{MH}]^{+}$, calcd for $\mathrm{C}_{16} \mathrm{H}_{22} \mathrm{NO}_{3} 276.1600$.

6: Pale yellow gum; IR $v_{\max } 2967,2914,1787,1664,1021$, $967 \mathrm{~cm}^{-1} ;[\alpha]_{D}^{25}+8\left(c 1.6, \mathrm{CHCl}_{3}\right) ;{ }^{1} \mathrm{H}$ NMR $\delta 6.80(\mathrm{~d}, J=16.0$ $\mathrm{Hz}, 1 \mathrm{H}, \mathrm{H}-1^{\prime}$ ), 6.39 (d, J = $16.0 \mathrm{~Hz}, 1 \mathrm{H}, \mathrm{H}-2^{\prime}$ ), 4.40 (br s, 1H, H2), 3.53 (br s, 1H, H-9a), 3.15-3.04 (m, 2H, H-5), 2.94 (d, $J=$ $5.0 \mathrm{~Hz}, 1 \mathrm{H}, \mathrm{H}-7), 2.79$ (dq, 1H, $J=12.0,7.0, \mathrm{H}-10), 2.29$ (s, 3H, H-4') 2.04-1.98 (m, 1H, H-1a), 2.01-1.96 (m, 1H, H-9), 1.88-1.82 (m, 2H, H-6), 1.85-1.79 (m, 1H, H-1b), 1.29 (d, $J=$ $6.5 \mathrm{~Hz}, 3 \mathrm{H}, \mathrm{H}-12) ;{ }^{13} \mathrm{C}$ NMR $\delta 198.0\left(\mathrm{C}-3^{\prime}\right), 177.9$ (C-11), 143.5 (C-1'), 130.7 (C-2'), 109.2 (C-8), 83.5 (C-3), 80.3 (C-2), 61.3 (C9a), 52.6 (C-7), 45.7 (C-9), 48.3 (C-5), 35.9 (C-10), 32.3 (C-1), 27.9 (C-4'), 26.7 (C-6), 13.3 (C-12); HRESIMS m/z 290.1389 $[\mathrm{MH}]^{+}$, calcd for $\mathrm{C}_{16} \mathrm{H}_{20} \mathrm{NO}_{4} 290.1392$.

\subsubsection{Synthesis of 6 by oxidation of ketone 5}

This was achieved by the general procedure using 5 (6.9 $\mathrm{mg}$, $0.025 \mathrm{mmol}), n$-butyl butanoate $(1 \mathrm{~mL})$, DIB (64.4mg, 8 equiv., $0.20 \mathrm{mmol}), \mathrm{Mg}(\mathrm{OAc})_{2} \bullet 4 \mathrm{H}_{2} \mathrm{O}(5.4 \mathrm{mg}, 1$ equiv., $0.025 \mathrm{mmol})$ and TBHP (120.0 $\mu \mathrm{L}, 24$ equiv., $0.60 \mathrm{mmol})$ to give $2.6 \mathrm{mg}$ $(42 \%)$ of enone 6 after column chromatography using gradient elution from $100 \%$ EtOAc to $\mathrm{MeOH} / \mathrm{EtOAc}(1: 4)$. 


\subsubsection{Oxidation of stemofoline 7}

This was achieved by the general procedure using $7(19.3 \mathrm{mg}$, $0.05 \mathrm{mmol}), n$-butyl butanoate $(1 \mathrm{~mL}), \mathrm{DIB}(16.1 \mathrm{mg}, 0.05$ mmol), $\mathrm{Mg}(\mathrm{OAc})_{2} \bullet 4 \mathrm{H}_{2} \mathrm{O}(10.7 \mathrm{mg}, 0.05 \mathrm{mmol})$ and TBHP (30.0 $\mu \mathrm{L}, 0.15 \mathrm{mmol})$ to give $8.0 \mathrm{mg}(58 \%)$ of the known lactone $\mathbf{8}$, after column chromatography using gradient elution from $100 \%$ EtOAc to $\mathrm{MeOH} / \mathrm{EtOAc}$ (1:4). The product was spectroscopically identical to that reported. ${ }^{2}$

\subsubsection{4-Oxohex-5-enyl benzoate (9a).}

The title compound was obtained using the general procedure described above from $9(22.2 \mathrm{mg}, 0.11 \mathrm{mmol}), n$-butyl butanoate $(1 \mathrm{~mL}), \mathrm{DIB}(34.8 \mathrm{mg}, 0.11 \mathrm{mmol}), \mathrm{Mg}(\mathrm{OAc})_{2} \bullet 4 \mathrm{H}_{2} \mathrm{O}(23.2 \mathrm{mg}$, $0.11 \mathrm{mmol})$ and TBHP $(64.8 \mu \mathrm{L}, 0.33 \mathrm{mmol})$. Purification by column chromatography using EtOAc/ether (15:85) as eluent gave $16.9 \mathrm{mg}$ (72\%) of 9a. Colourless oil; IR $v_{\max } 2959,2902$, $1716,1675,1270,1110 \mathrm{~cm}^{-1} ;{ }^{1} \mathrm{H}$ NMR $\delta 8.03(\mathrm{~d}, J=7.0 \mathrm{~Hz}, 2 \mathrm{H}$, ArH2 and 6), 7.56 (t, $J=7.0 \mathrm{~Hz}, 1 \mathrm{H}, \mathrm{ArH} 4), 7.44(\mathrm{t}, J=7.0 \mathrm{~Hz}$, $2 \mathrm{H}, \mathrm{ArH} 3$ and 5), 6.38 (dd, $\left.J=17.5,7.5 \mathrm{~Hz}, 1 \mathrm{H}, \mathrm{H} 5^{\prime}\right), 6.25(\mathrm{~d}, J$ $\left.=17.5 \mathrm{~Hz}, 1 \mathrm{H}, \mathrm{H} 6^{\prime} \mathrm{a}\right), 5.85\left(\mathrm{~d}, J=7.5 \mathrm{~Hz}, 1 \mathrm{H}, \mathrm{H} 6^{\prime} \mathrm{b}\right), 4.37$ (t, $J=$ $\left.7.0 \mathrm{~Hz}, 2 \mathrm{H}, \mathrm{H} 1^{\prime}\right), 2.77$ (t, $\left.J=7.0 \mathrm{~Hz}, 2 \mathrm{H}, \mathrm{H} 3^{\prime}\right), 2.15-2.12(\mathrm{~m}$, $\left.2 \mathrm{H}, \mathrm{H} 2{ }^{\prime}\right) ;{ }^{13} \mathrm{C}$ NMR $\delta 199.7\left(\mathrm{C}^{\prime}\right), 166.7$ (COO), $136.6\left(\mathrm{C}^{\prime}\right)$, 133.1 (ArC4), 129.7 (ArC2 and 6), $128.6\left(\mathrm{C}^{\prime}\right), 128.5$ (ArC1, 3 and 5), $64.3\left(\mathrm{C}^{\prime}\right), 36.1\left(\mathrm{C}^{\prime}\right), 23.2\left(\mathrm{C}^{\prime}\right)$; HRESIMS $\mathrm{m} / \mathrm{z}$ 219.1028 [MH] $]^{+}$, calcd for $\mathrm{C}_{13} \mathrm{H}_{15} \mathrm{O}_{3} 219.1021$.

\subsubsection{8-Oxodec-9-enyl benzoate (10a)}

The title compound was obtained using the general procedure described above from $\mathbf{1 0}(32.8 \mathrm{mg}, 0.13 \mathrm{mmol}), n$-butyl butanoate $(1 \mathrm{~mL}), \mathrm{DIB}(40.6 \mathrm{mg}, 0.13 \mathrm{mmol}), \mathrm{Mg}(\mathrm{OAc}) 2 \bullet 4 \mathrm{H}_{2} \mathrm{O}$ (27.0 $\mathrm{mg}, 0.13 \mathrm{mmol}$ ) and TBHP $(75.6 \mu \mathrm{L}, 0.39 \mathrm{mmol})$. Purification by column chromatography using gradient elution from $100 \%$ ether to EtOAc/ether (1:1) gave $15.7 \mathrm{mg}(46 \%)$ of 10a. Colourless oil; IR $v_{\max } 2931,2859,1718,1271,1112 \mathrm{~cm}^{-1}$; ${ }^{1} \mathrm{H}$ NMR $\delta 7.97(\mathrm{~d}, J=7.5 \mathrm{~Hz}, 2 \mathrm{H}, \mathrm{ArH} 2$ and 6), $7.48(\mathrm{t}, J=7.5$ $\mathrm{Hz}, 1 \mathrm{H}, \mathrm{ArH} 4), 7.37$ (t, $J=7.5 \mathrm{~Hz}, 2 \mathrm{H}, \mathrm{ArH} 3$ and 5), 6.28 (dd, $J$ $\left.=17.5,10.5 \mathrm{~Hz}, 1 \mathrm{H}, \mathrm{H}^{\prime}\right), 6.14\left(\mathrm{~d}, J=17.5 \mathrm{~Hz}, 1 \mathrm{H}, \mathrm{H} 10^{\prime} \mathrm{a}\right), 5.74$ $\left(\mathrm{d}, J=10.5 \mathrm{~Hz}, 1 \mathrm{H}, \mathrm{H} 10^{\prime} \mathrm{b}\right), 4.24\left(\mathrm{t}, J=7.0 \mathrm{~Hz}, 2 \mathrm{H}, \mathrm{H} 1^{\prime}\right), 2.51(\mathrm{t}$, $\left.J=7.0 \mathrm{~Hz}, 2 \mathrm{H}, \mathrm{H} 7^{\prime}\right), 1.70$ (tt, $\left.J=7.0,7.0 \mathrm{~Hz}, 2 \mathrm{H}, \mathrm{H} 2^{\prime}\right), 1.57$ (tt, $\left.J=7.0,7.0 \mathrm{~Hz}, 2 \mathrm{H}, \mathrm{H}^{\prime}\right), 1.41-1.38\left(\mathrm{~m}, 2 \mathrm{H}, \mathrm{H}^{\prime}\right), 1.34-1.25(\mathrm{~m}$, 4H, H4' and $\left.5^{\prime}\right) ;{ }^{13} \mathrm{C}$ NMR $\delta 201.1$ (C8'), 166.8 (COO), 136.7 (C9'), 132.9 (ArC4), 130.6 (ArC1), 129.7 (ArC2 and 6), 128.5

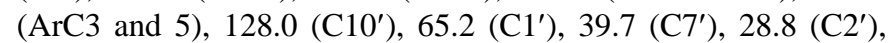
$29.2\left(\mathrm{C}^{\prime}\right.$ and $\left.5^{\prime}\right), 26.0\left(\mathrm{C}^{\prime}\right), 24.0\left(\mathrm{C}^{\prime}\right)$; HRESIMS $\mathrm{m} / \mathrm{z}$ 275.1647 [MH] $]^{+}$, calcd for $\mathrm{C}_{17} \mathrm{H}_{23} \mathrm{O}_{3} 275.1647$.

\subsection{6. (E)-3-Oxohex-4-enyl benzoate (11a)}

The title compound was obtained using the general procedure described above from $11(52.8 \mathrm{mg}, 0.26 \mathrm{mmol}), n$-butyl butanoate $(1 \mathrm{~mL}), \mathrm{DIB}(83.8 \mathrm{mg}, 0.26 \mathrm{mmol}), \mathrm{Mg}(\mathrm{OAc})_{2} \bullet 4 \mathrm{H}_{2} \mathrm{O}$ (55.8 mg, $0.26 \mathrm{mmol}$ ) and TBHP (156.0 $\mu \mathrm{L}, 0.78 \mathrm{mmol})$. Purification by column chromatography using gradient elution from $100 \%$ ether to EtOAc/ether (1:1) gave $12.0 \mathrm{mg}$ (21\%) of 9a, $8.4 \mathrm{mg}$ of 11a (15\%) and $15.4 \mathrm{mg}$ of $\mathbf{1 1 b}(27 \%)$. Compound 11b was spectroscopically identical to that reported. ${ }^{12}$ 11a: colourless oil. ${ }^{1} \mathrm{H}$ NMR $\delta 8.00(\mathrm{~d}, J=7.0 \mathrm{~Hz}, 2 \mathrm{H}, \mathrm{ArH} 2$ and 6$), 7.55(\mathrm{t}, J=$ $7.0 \mathrm{~Hz}, 1 \mathrm{H}, \mathrm{ArH} 4), 7.43(\mathrm{t}, J=7.0 \mathrm{~Hz}, 2 \mathrm{H}, \mathrm{ArH} 3$ and 5), 6.91 $\left(\mathrm{dq}, J=15.0,7.0 \mathrm{~Hz}, 1 \mathrm{H}, \mathrm{H} 5^{\prime}\right), 6.18\left(\mathrm{~d}, J=15.0 \mathrm{~Hz}, 1 \mathrm{H}, \mathrm{H} 4^{\prime}\right)$, $4.64\left(\mathrm{t}, J=7.0 \mathrm{~Hz}, 2 \mathrm{H}, \mathrm{H} 1^{\prime}\right), 3.01\left(\mathrm{tt}, J=7.0,7.0 \mathrm{~Hz}, 2 \mathrm{H}, \mathrm{H} 2^{\prime}\right.$ ), $1.92\left(\mathrm{~d}, J=7.0 \mathrm{~Hz}, 3 \mathrm{H}, \mathrm{H}^{\prime}\right) ;{ }^{13} \mathrm{C}$ NMR $\delta 197.2\left(\mathrm{C}^{\prime}\right), 166.6$ (COO), 144.0 (C5'), 132.1 (ArC4 and $\mathrm{C4}^{\prime}$ ), 129.7 (ArC2 and 6), 128.5 (ArC1, 3 and 5), $60.4\left(\mathrm{C}^{\prime}\right), 38.7\left(\mathrm{C} 2^{\prime}\right), 18.5\left(\mathrm{C}^{\prime}\right)$; HRESIMS $m / z 219.1029[\mathrm{MH}]^{+}$, calcd for $\mathrm{C}_{13} \mathrm{H}_{15} \mathrm{O}_{3} 219.1021$.

\subsubsection{Oxidation of benzyl-5-hexenyl ether $\mathbf{1 2}$}

This was achieved by the general procedure using 12 (42.2 $\mathrm{mg}, 0.22 \mathrm{mmol}$ ), $n$-butyl butanoate (l mL), DIB (70.9 mg, 0.22 $\mathrm{mmol}), \mathrm{Mg}(\mathrm{OAc}) 2 \cdot 4 \mathrm{H} 2 \mathrm{O}(47.2 \mathrm{mg}, 0.22 \mathrm{mmol})$ and TBHP $(132.0 \mu \mathrm{L}, 0.66 \mathrm{mmol})$ to give $27.2 \mathrm{mg}(61 \%)$ of $\mathbf{9}, 7.8 \mathrm{mg}$ of $\mathbf{9 a}$ (16\%) and $0.9 \mathrm{mg}$ of 12a (2\%) after column chromatography using gradient elution from $100 \%$ ether to EtOAc/ether (1:9), Compound 12a was spectroscopically identical to that reported. ${ }^{13}$

\subsubsection{3-Phenylpropyl acrylate $\mathbf{1 3 a}$}

The title compound was obtained using the general procedure described above from 13 (107.7 mg, $0.61 \mathrm{mmol})$, ethyl acetate (1 $\mathrm{mL}$ ), DIB (296.3 mg, 1.5 equiv., $0.92 \mathrm{mmol}), \mathrm{Mg}(\mathrm{OAc})_{2} \bullet 4 \mathrm{H}_{2} \mathrm{O}$ (130.8 mg, $0.61 \mathrm{mmol})$ and TBHP $(549.0 \mu \mathrm{L}, 4.5$ equiv., 2.75 mmol) to give $104.2 \mathrm{mg}(90 \%)$ of $\mathbf{1 3 a}$ after column chromatography using gradient elution from $100 \%$ ether to EtOAc/ether (1:9). Compound 13a was spectroscopically identical to that reported. ${ }^{14}$

\subsubsection{3-Phenylpropyl benzoate 14a}

The title compound was obtained using the general procedure described above from 14 (102.7 $\mathrm{mg}, 0.45 \mathrm{mmol})$, ethyl acetate (1 $\mathrm{mL}$ ), DIB (217.4 mg, 1.5 equiv., $0.68 \mathrm{mmol}), \mathrm{Mg}(\mathrm{OAc})_{2} \bullet 4 \mathrm{H}_{2} \mathrm{O}$ (96.5 mg, $0.45 \mathrm{mmol})$ and TBHP $(405.0 \mu \mathrm{L}, 4.5$ equiv., 2.03 mmol) to give $102.5 \mathrm{mg}(95 \%)$ of $\mathbf{1 4 a}$ after column chromatography using gradient elution from $100 \%$ ether to EtOAc/ether (1:9). Compound 14a was spectroscopically identical to that reported. ${ }^{15}$

\subsubsection{Hexyl benzoate 15a}

The title compound was obtained using the general procedure described above from $15(108.8 \mathrm{mg}, 0.57 \mathrm{mmol})$, ethyl acetate (1 $\mathrm{mL})$, DIB (277.0 mg, 1.5 equiv., $0.86 \mathrm{mmol}), \mathrm{Mg}(\mathrm{OAc})_{2} \bullet 4 \mathrm{H}_{2} \mathrm{O}$ (122.3 mg, $0.57 \mathrm{mmol})$ and TBHP $(684.0 \mu \mathrm{L}, 6.0$ equiv., 3.42 $\mathrm{mmol})$ to give $109.2 \mathrm{mg}(93 \%)$ of $\mathbf{1 5 a}$ after column chromatography using gradient elution from $100 \%$ ether to EtOAc/ether (1:9). Compound 15a was spectroscopically identical to that reported. ${ }^{16}$

\subsubsection{Hexyl acrylate $16 a$}

The title compound was obtained using the general procedure described above from 16 (70.3 $\mathrm{mg}, 0.50 \mathrm{mmol})$, ethyl acetate (1 $\mathrm{mL}$ ), DIB (241.6 mg, 1.5 equiv., $0.75 \mathrm{mmol}), \mathrm{Mg}(\mathrm{OAc})_{2} \bullet 4 \mathrm{H}_{2} \mathrm{O}$ (107.2 mg, $0.50 \mathrm{mmol})$ and TBHP $(600.0 \mu \mathrm{L}, 6.0$ equiv., 3.0 mmol) to give $71.0 \mathrm{mg}(91 \%)$ of $\mathbf{1 6 a}$ after column chromatography using gradient elution from $100 \%$ ether to EtOAc/ether (1:9). Compound 16a was spectroscopically identical to that reported. ${ }^{17}$

\subsubsection{Hex-5-enyl acrylate (17a)}

The title compound was obtained using the general procedure described above from 17 (106.8 mg, $0.76 \mathrm{mmol})$, ethyl acetate (1 $\mathrm{mL}$ ), DIB (367.2 mg, 1.5 equiv., $1.14 \mathrm{mmol}), \mathrm{Mg}(\mathrm{OAc})_{2} \bullet 4 \mathrm{H}_{2} \mathrm{O}$ (163.0 mg, $0.76 \mathrm{mmol})$ and TBHP $(912.0 \mu \mathrm{L}, 6.0$ equiv., 4.56 mmol). Purification by column chromatography using gradient elution from $100 \%$ ether to EtOAc/ether $(1: 1)$ gave $103.2 \mathrm{mg}$ $(88 \%)$ of 17a. Colourless oil; IR $v_{\max } 2959,2902,1716,1675$, $1270,1110 \mathrm{~cm}^{-1}$; ${ }^{1} \mathrm{H}$ NMR $\delta 6.40(\mathrm{~d}, J=17.5,1 \mathrm{H}, \mathrm{H} 1 \mathrm{a}), 6.12(\mathrm{dd}$, $J=17.5,10.5,1 \mathrm{H}, \mathrm{H} 2), 5.82(\mathrm{~d}, J=10.5,1 \mathrm{H}, \mathrm{H} 1 \mathrm{~b}), 5.83-5.76$ (m, 1H, H5'), 5.02 (d, $\left.J=17.0,1 \mathrm{H}, \mathrm{H} 66^{\prime} \mathrm{a}\right), 4.97$ (d, $J=10.0 \mathrm{~Hz}$, $\left.1 \mathrm{H}, \mathrm{H}^{\prime} \mathrm{b}\right), 4.16\left(\mathrm{t}, J=7.0 \mathrm{~Hz}, 1 \mathrm{H}, \mathrm{H}^{\prime}\right), 2.09(\mathrm{dt}, J=7.0,7.0 \mathrm{~Hz}$, $\left.1 \mathrm{H}, \mathrm{H} 4^{\prime}\right), 1.69\left(\mathrm{tt}, J=7.0,7.0 \mathrm{~Hz}, 1 \mathrm{H}, \mathrm{H} 2^{\prime}\right), 1.48(\mathrm{tt}, J=7.0,7.0$ $\left.\mathrm{Hz}, 1 \mathrm{H}, \mathrm{H} 3^{\prime}\right) ;{ }^{13} \mathrm{C}$ NMR $\delta 166.7$ (COO), 138.5 (C5), 130.6 (C1), 
$128.4(\mathrm{C} 2), 115.0\left(\mathrm{C}^{\prime}\right), 64.6\left(\mathrm{C}^{\prime}\right), 33.4\left(\mathrm{C}^{\prime}\right), 28.1\left(\mathrm{C}^{\prime}\right), 25.3$ $\left(\mathrm{C}^{\prime}\right)$; HRESIMS $\mathrm{m} / \mathrm{z} 155.1439[\mathrm{MH}]^{+}$, calcd for $\mathrm{C}_{9} \mathrm{H}_{15} \mathrm{O}_{2}$ 155.1072 .

4.2.13. 4-(Allyloxy) butyl benzoate (18a), 4-(benzyloxy) butyl acrylate $(\mathbf{1 8 b})$ and 4-(acryloxy) butyl benzoate (18c)

The title compounds were obtained using the general procedure described above from $18(220.3 \mathrm{mg}, 1.0 \mathrm{mmol})$, ethyl acetate $(1 \mathrm{~mL})$, DIB $(483.2 \mathrm{mg}, 1.5$ equiv., $1.5 \mathrm{mmol})$, $\mathrm{Mg}(\mathrm{OAc})_{2} \bullet 4 \mathrm{H}_{2} \mathrm{O}(214.5 \mathrm{mg}, 1.0 \mathrm{mmol})$ and TBHP $(1,200.0 \mu \mathrm{L}$, 6.0 equiv., $6.0 \mathrm{mmol}$ ). Purification by column chromatography using gradient elution from $100 \%$ ether to EtOAc/ether.(1:1) gave $84.2 \mathrm{mg}(25 \%)$ of $\mathbf{1 8 a}, 32.8 \mathrm{mg}$ of $\mathbf{1 8 b}(25 \%)$ and $99.2 \mathrm{mg}$ of $18 \mathbf{c}(40 \%)$.

18a: Colourless oil; IR $v_{\max }$ 2959, 2902, 1716, 1675, 1270, $1110 \mathrm{~cm}^{-1} ;{ }^{1} \mathrm{H}$ NMR $\delta 8.04(\mathrm{~d}, J=7.5 \mathrm{~Hz}, 2 \mathrm{H}, \mathrm{ArH} 2$ and 6$), 7.55$ (t, $J=7.5 \mathrm{~Hz}, 1 \mathrm{H}, \mathrm{ArH} 4), 7.43$ (t, $J=7.5 \mathrm{~Hz}, 2 \mathrm{H}, \mathrm{ArH} 3$ and 5), 5.96-5.87 (m, 1H, H7'), 5.28 (d, $\left.J=17.5 \mathrm{~Hz}, 1 \mathrm{H}, \mathrm{H} 8^{\prime} \mathrm{a}\right), 5.17$ (d, $\left.J=10.0 \mathrm{~Hz}, 1 \mathrm{H}, \mathrm{H} 8^{\prime} \mathrm{b}\right), 4.35\left(\mathrm{t}, J=7.0 \mathrm{~Hz}, 2 \mathrm{H}, \mathrm{H} 1^{\prime}\right), 3.98$ (d, $J=$ $\left.5.5 \mathrm{~Hz}, 2 \mathrm{H}, \mathrm{H6}^{\prime}\right), 3.50\left(\mathrm{t}, J=6.0 \mathrm{~Hz}, 2 \mathrm{H}, \mathrm{H} 4^{\prime}\right), 1.87$ (tt, $J=7.0$, $\left.6.5 \mathrm{~Hz}, 2 \mathrm{H}, \mathrm{H} 2^{\prime}\right), 1.77\left(\mathrm{tt}, J=7.0,6.0 \mathrm{~Hz}, 2 \mathrm{H}, \mathrm{H} 3{ }^{\prime}\right) ;{ }^{13} \mathrm{C} \mathrm{NMR} \delta$ 166.8 (COO), 135.1 (C7'), 132.9 (ArC4), 130.6 (ArC1), 129.7 (ArC2 and 6), 128.5 (ArC3 and 5), 116.8 (C8'), 71.8 (C6'), 70.0 (C4'), $64.8\left(\mathrm{C} 1^{\prime}\right), 26.4\left(\mathrm{C} 3^{\prime}\right), 25.6\left(\mathrm{C}^{\prime}\right)$; HRESIMS $\mathrm{m} / z, 235$ $[\mathrm{MH}]^{+}$, calcd for $\mathrm{C}_{14} \mathrm{H}_{19} \mathrm{O}_{3} 235$.

18b: Colourless oil; IR $v_{\max } 2959,2902,1716,1675,1270$, $1110 \mathrm{~cm}^{-1} ;{ }^{1} \mathrm{H}$ NMR $\delta 7.58-7.54(\mathrm{~m}, 2 \mathrm{H}, \mathrm{ArH} 2$ and 6), $7.47-7.41(\mathrm{~m}, 1 \mathrm{H}, \mathrm{ArH} 3,4$ and 5), $6.39(\mathrm{~d}, J=17.5 \mathrm{~Hz}, 1 \mathrm{H}$, H8'a), 6.15-6.08 (m, 1H, H7'), 5.80 (d, $\left.J=10.0 \mathrm{~Hz}, 1 \mathrm{H}, \mathrm{H} 8^{\prime} \mathrm{b}\right)$, 4.51 (br. s, $\left.2 \mathrm{H}, P h-\mathrm{CH}_{2}-\mathrm{O}\right), 4.18\left(\mathrm{t}, J=6.0 \mathrm{~Hz}, 2 \mathrm{H}, \mathrm{H} 4^{\prime}\right), 3.51$ (t, $\left.J=6.0 \mathrm{~Hz}, 2 \mathrm{H}, \mathrm{H} 1^{\prime}\right), 1.78\left(\mathrm{tt}, J=7.0,6.0 \mathrm{~Hz}, 2 \mathrm{H}, \mathrm{H} 3^{\prime}\right), 1.71(\mathrm{tt}$, $\left.J=7.0,6.0 \mathrm{~Hz}, 2 \mathrm{H}, \mathrm{H} 22^{\prime}\right) ;{ }^{13} \mathrm{C} \mathrm{NMR} \delta 166.5$ (COO), 137.5 (ArC1), 133.0 (ArC2 and 6), $130.9\left(\mathrm{C}^{\prime}\right), 128.6\left(\mathrm{C}^{\prime}\right), 128.5$ (ArC3, 4 and 5), 73.1 (Ph- $\left.\mathrm{CH}_{2}-\mathrm{O}\right), 69.8\left(\mathrm{C}^{\prime}\right), 64.4\left(\mathrm{C}^{\prime}{ }^{\prime}\right), 26.3$ $\left(\mathrm{C}^{\prime}\right), 25.8\left(\mathrm{C}^{\prime}\right)$; HRESIMS $\mathrm{m} / z 235[\mathrm{MH}]^{+}$, calcd for $\mathrm{C}_{14} \mathrm{H}_{19} \mathrm{O}_{3}$ 235.

18c: Light brown oil; IR $v_{\max } 2959,2902,1716,1675,1270$, $1110 \mathrm{~cm}^{-1} ;{ }^{1} \mathrm{H}$ NMR $\delta 8.04(\mathrm{~d}, J=7.5 \mathrm{~Hz}, 2 \mathrm{H}, \mathrm{ArH} 2$ and 6$), 7.55$ (t, $J=7.5 \mathrm{~Hz}, 1 \mathrm{H}, \mathrm{ArH} 4), 7.44(\mathrm{t}, J=7.5 \mathrm{~Hz}, 2 \mathrm{H}, \mathrm{ArH} 3$ and 5), $6.41\left(\mathrm{~d}, J=17.5 \mathrm{~Hz}, 1 \mathrm{H}, \mathrm{H} 8^{\prime} \mathrm{a}\right), 6.13(\mathrm{dd}, J=17.5,10.0,1 \mathrm{H}$, H7'), $5.82\left(\mathrm{~d}, J=10.0 \mathrm{~Hz}, 1 \mathrm{H}, \mathrm{H} 8^{\prime} \mathrm{b}\right), 4.37(\mathrm{t}, J=6.0 \mathrm{~Hz}, 2 \mathrm{H}$, $\left.\mathrm{H} 1^{\prime}\right), 4.24\left(\mathrm{t}, J=6.0 \mathrm{~Hz}, 2 \mathrm{H}, \mathrm{H} 4^{\prime}\right), 1.92-1.82\left(\mathrm{~m}, 2 \mathrm{H}, \mathrm{H} 2^{\prime}\right)$, $1.91-1.81\left(\mathrm{~m}, 2 \mathrm{H}, \mathrm{H} 3^{\prime}\right) ;{ }^{13} \mathrm{C}$ NMR $\delta 166.7$ (COO), 166.3 (C6'), 133.1 (ArC4), 130.9 (C8'), 130.4 (ArC1), 129.7 (ArC2 and 6), 128.5 ( $\mathrm{C}^{\prime}$ and $\mathrm{ArC} 3$ and 5), 64.5 ( $\mathrm{C} 1^{\prime}$ and $\left.4^{\prime}\right), 25.5$ (C2' and $\left.3^{\prime}\right)$; HRESIMS $m / z$ 249.1132 $[\mathrm{MH}]^{+}$, calcd for $\mathrm{C}_{14} \mathrm{H}_{17} \mathrm{O}_{4}$ 249.1127

\section{Acknowledgments}

We thank the Australian Research Council and the University of Wollongong for financial support.

\section{References and notes}

1. Zhao, Y.; Yeung, Y.-Y. Org. Lett. 2010, 12, 2128-2131.

2. Sastraruji, K.; Sastraruji, T.; Pyne, S. G.; Ung, A. T.; Jatisatienr, A.; Lie, W. J. Nat. Prod. 2010, 73, 935-941.

3. Zhao, Y.; Yim, W.-L.; Tan, C. K.; Yeung; Y.-Y. Org. Lett. 2011, 13, 4308-4311.

4. Sastraruji, T.; Jatisatienr, A.; Pyne, S. G.; Ung, A. T.; Lie, W.; Williams, M. C. J. Nat. Prod. 2005, 68, 1763-1767.

5. Schleicher, K. D.; Jamison, T. F. Org. Lett. 2007, 9, 875-878

6. Rawat, V.; Chouthaiwale, P. V.; Suryavanshi, G.; Sudalai, A. Tetrahedron: Asymmetry 2009, 20, 2173-2177.

7. Srihari, P.; Bhasker, E. V.; Harshavardhan, S. J.; Yadav, J. S. Synthesis 2006, 23, 4041-4045.

8. Ohkubo, M.; Mochizuki, S.; Sano, T.; Kawaguchi, Y.; Okamoto, S. Org. Lett. 2007, 9, 773-776.

9. Kurita, T.; Hattori, K.; Seki, S.; Mizumoto, T.; Aoki, F.; Yamada, Y.; Ikawa, K.; Maegawa, T.; Monguchi, Y.; Sajiki, H. Chem. Eur J. 2008, 14, 664-673.

10. Chu, Q; Brahmi, M. M.; Solovyev, A.; Ueng, S.; Curran, D. P.; Malacria, M.; Fensterbank, L.; Lacote, E., Chem. Eur. J. 2009,15, 12937-12940.

11. Su, C.; Williard, P. G. Org. Lett. 2010, 12, 5378-5381.

12. Clavier, H.; Caijo, F.; Borre, E.; Rix, D.; Boeda, F.; Nolan, S. P.; Mauduit, M. Eur. J. Org. Chem.2009, 25, 4254-4265.

13. Iyengar, R.; Schildknegt, K.; Morton, M.; Aube, J. J. Org. Chem. 2005, 70, 10645-10652.

14. Kim, J.; Park, E.; Shim, J.; Kim, M.; Moon, W.; Chung, K.; Yoon, J. J. Agric. Food Chem. 2004, 52, 7480-7483.

15. Werner, T.; Barrett, Anthony G. M. J. Org. Chem. 2006, 71, 4302-4304.

16. Won, J.; Kim, H.; Kim, J.; Yim, H.; Kim, M.; Kang, S.; Chung, H..; Lee, S.; Yoon, Y. Tetrahedron 2007, 63, 12720-12730.

17. AIST: Integrated Spectral Database System of Organic Compounds. (Data were obtained from the National Institute of Advanced Industrial Science and Technology (Japan))

\section{Supplementary Material}

Copies of the ${ }^{1} \mathrm{H}$ NMR spectra of all compounds. This material is available free of charge via the internet at. 